\title{
Fabrication of Bragg gratings on the End Facet of Standard Optical Fibers by Sputtering the Same Material
}

\author{
Joaquín Ascorbe, Jesus M. Corres, Ignacio del Villar, Francisco J. Arregui, Member, IEEE, and \\ Ignacio R. Matias, Senior Member, IEEE
}

\begin{abstract}
A sputtering process has been applied to deposit quarter-wavelength stacks on the end facet of cleaved optical fibers by using only one material. Standard multimode optical fibers were used as substrates to fabricate broadband filters, and the experimentally measured spectral responses of these devices are shown. Periodical changes in the refractive index of the coating have been achieved by changing the sputtering pressure. A reflected peak with a full width at half maximum of $20 \mathrm{~nm}$ centered at $440 \mathrm{~nm}$ has been obtained, which provides a good structure for the development of optical fiber sensors working with the wavelength detection technique. This optical structure can be used for several purposes: as tunable wavelength filters or optical fiber sensors or to improve the performance of fluorescence sensors. A theoretical analysis of these structures corroborates the experimental results and allows some rules to be obtained.
\end{abstract}

Index Terms - fiber optics components, interference coatings, Bragg gratings, tunable filters, band-pass filters, sputtering

\section{INTRODUCTION}

$\mathrm{T}_{\mathrm{s}}$ HE sputtering technique is a well-known method used to build up coatings on a variety of different substrate materials such as ceramics or metals. These thin films have been extensively used for the fabrication of electronic devices, optical coatings, instrument hard coatings, and decorative parts [1]. The fine tuning of the sputtering condition (power, current, vacuum pressure, gas flow rate, etc.) allows one to have excellent control over the thickness of the deposited films, making sputtering a useful tool for depositing coatings on optical fibers [2]-[5]. In fact, sputtering has been used to fabricate nano-Fabry-Perot interferometers on the end facet of optical fibers in order to develop devices for different sensing

Manuscript submitted September 27, 2016. This work was supported by a Public University of Navarra PhD Grant and by the Spanish Economy and Competitiveness Ministry, TEC-2013-43679-R.

Joaquin Ascorbe is with the Department of Electrical and Electronic Engineering, Public University of Navarra.

All other authors are with the Department of Electrical and Electronic Engineering and the Institute of Smart Cities, Public University of Navarra, 31006 Pamplona, Spain (e-mail: joaquin.ascorbe@unavarra.es; jmcorres@unavarra.es; ignacio.delvillar@unavarra.es; parregui@unavarra.es; natxo@unavarra.es). purposes such as relative humidity sensing [6] or gas detection [7].

Multilayer coatings can be used for several applications, such as band-pass filters [8], antireflective coatings [8], [9], temperature sensors [10], [11], humidity sensors [12], and others [13]-[17]. Traditionally, these devices require at least two different materials for their fabrication. This can present some advantages such as providing a reference wavelength [13], but it can be disadvantageous in other situations. The utilization of two or more materials is generally timeconsuming. In addition to this, the behavior of these interference filters might depend on the wavelength range selected for performing the measurements [11], especially when they are developed with materials that have great differences in their refractive indices (RIs). Besides, filters developed with a few bilayers present low wavelength resolution [13] due to having a poorly defined reflected peak.

In previous works, it has been demonstrated that changes in the sputtering parameters can modify the optical constants of the thin film obtained [18], [19]. There are several causes: different crystallographic orientations (affecting the RI), degree of crystallinity, carrier concentration, and stoichiometry (which affects the extinction coefficient) [19].

Here, only one material has been used to build up the Bragg grating on the end facet, which permits one to avoid the problems mentioned above. This leads to an efficient method of obtaining a tunable narrowband optical filter in an automated way.

The most common type of fiber Bragg grating (FBG) is based on the periodic perturbation of the RI of an optical fiber. This structure is typically obtained by exposure of the waveguide core to an intense optical interference pattern [20]. The most common method of developing a Bragg grating is by the phase mask technique [21], [22], which requires a highly precise setup. This type of FBG is sensitive to temperature or strain [23]. However, this structure must be coated with a different material in order to make it sensitive to other parameters [24]. The method explained here integrates both elements - the grating and the sensitive material-in one step, which simplifies the fabrication process. Furthermore, this grating makes it possible to work in the ultraviolet-visible (UV-Vis) range instead of the habitual range of Bragg 
gratings developed for working in the near-infrared (NIR) range.

Two different stacks of layers, one developed with indium oxide and the other one with tin oxide, have been studied for the development of these band-pass filters. Moreover, the reflected peak has been tuned at different wavelengths, proving the high versatility of this method.

The manuscript is organized as follows. In Section II, a theoretical analysis is done and the main parameters affecting the features of the final device are studied. Then, in Section III, the experimentally obtained devices and their characteristics are discussed. In Section IV, a comparison between the theoretical and experimental results is made. Finally, some concluding remarks are presented in Section IV.

\section{THEORETICAL ANALYSIS}

In this section, some simulated results are shown to help understand the main factors involving the Bragg gratings fabrication. The setup used henceforth is depicted in Fig. 1(a). A white halogen source (ANDO AQ-4303B) launches light into one multimode standard communications fiber (MMF) with core and cladding diameters of 62.5 and $125 \mu \mathrm{m}$, respectively, through a $2 \times 1$ (50:50) splitter. The reflected light from the end facet is collected by an HR4000 (OceanOptics) spectrometer.

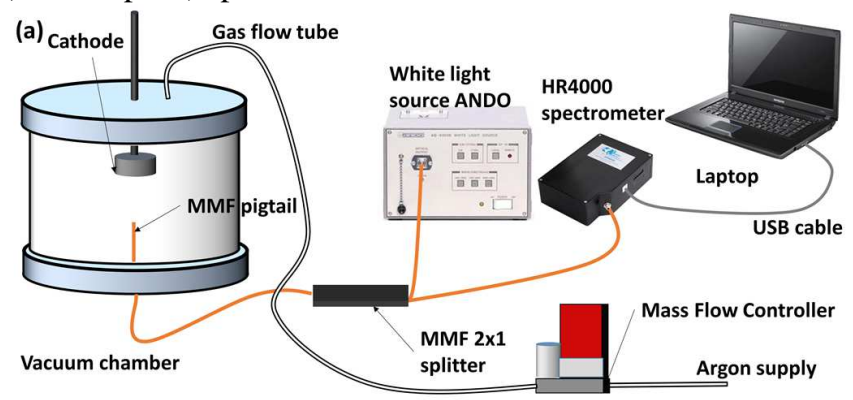

(b)

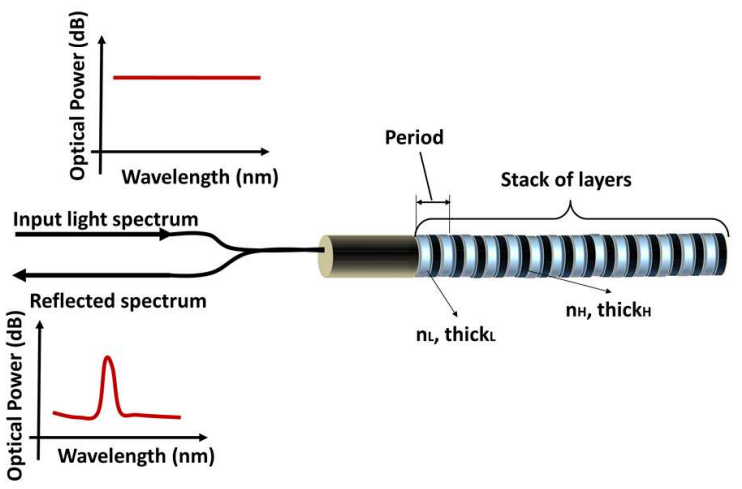

Fig. 1 (a) Experimental setup and (b) optical model.

Fig. 1(b) shows the optical model for the Bragg grating deposited on the multimode optical fiber end facet. Parameters such as the thickness and the number of periods were adequately dimensioned so that resonance could be obtained in the reflection spectrum, and the optical constants have been obtained from previous works [18], [19], [25], [26] to simulate transparent conductor oxides. These simulations have been performed with Matlab®, following a numerical method based on plane wave propagation in a one-dimensional stack of layers of different RIs [27], [28]. Each reflection spectrum with a stack of layers was normalized to the reflection spectrum without a stack of layers. Dispersion of the RI of the material was not taken into account in order to simplify the analysis of the obtained results.

The RI of the core of the optical fiber was set to 1.5 according to the data obtained from Telnet-RI, whereas the RI of the external medium was set to 1 . The RI of the low refractive index layer (LRIL) was assumed to be 1.7 , while the high refractive index layer (HRIL) was set as $10 \%$ greater than that of the LRIL, which is in the range obtained in [19]. Thicknesses for each layer were set to $80 \mathrm{~nm}$, which is a value easily obtained by sputtering. The particularities of the parameters of each situation under study are commented on in their respective sections. The main factors affecting this optical structure are the thickness of each layer, the number of periods of the thin-film stack, and the RI of each layer. The real and imaginary parts of the RI were studied separately.

\section{A. Difference between refractive indices}

First, the influence of the difference in the RI of each layer is shown in Fig. 2. These results were obtained using the following parameters: the RI of the even layers was set to 1.7 and the RI of the odd layers was varied from 1.7 to 1.8683 in $0.1 \%$ increments. Fig. 2 shows the simulated results for 50 periods.

\section{(a)}
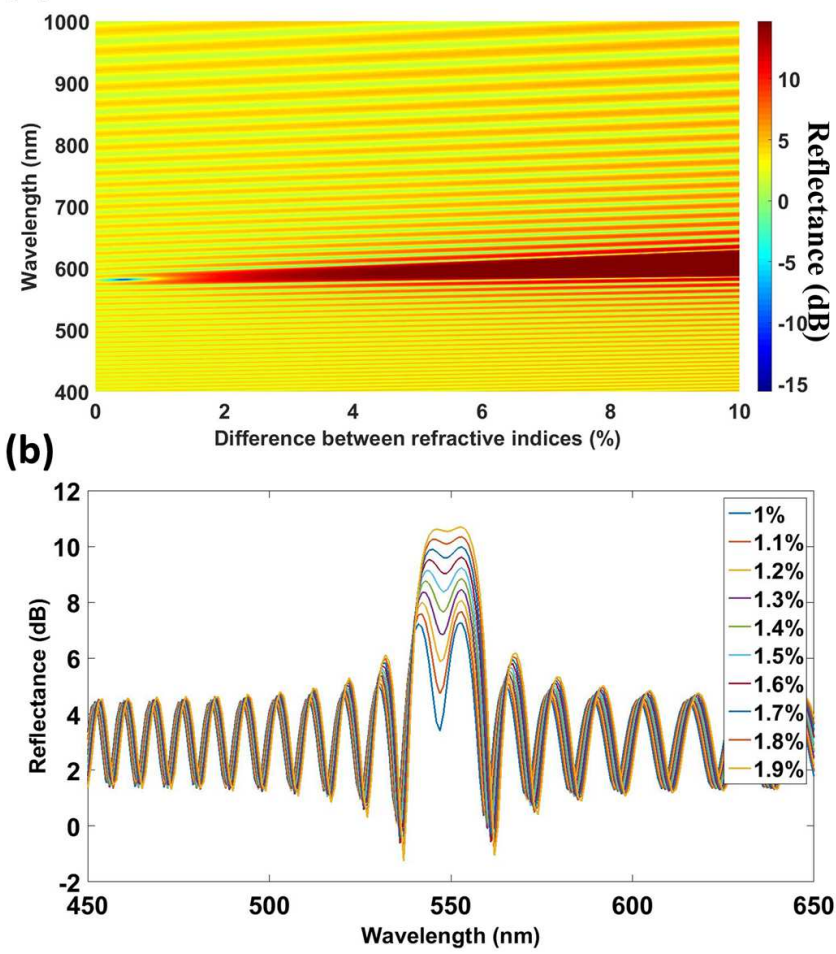

Fig. 2 (a) Evolution of the reflected spectrum for a difference in refractive indices ranging from 0 to $10 \%$; (b) spectra for different values of the difference between RIs. 
As can be seen in Fig. 2 (a), the width of the reflected peak increases with increases in the difference between the RIs. In the limiting case of both RIs being equal, we would obtain a Fabry-Pèrot interferometer. Differences of $2 \%$ are needed to have a well-defined peak (see Fig. 2(b)) with an amplitude of the reflected peak at least double that of the sidelobes. These percentage values might vary slightly with the number of periods. The reflected power depends on the difference between the RIs of each layer, tending asymptotically to its maximum of $14 \mathrm{~dB}$ for differences of $5 \%$.

\section{B. Number of periods}

The next parameter that was studied was the number of periods or bilayers $(\mathrm{N})$. Each period consists of two different layers (LRIL and HRIL). For these simulations, the RI of odd layers was set to 1.7 , the RI of even layers was set to 1.87 , and the thickness of each layer was set to $80 \mathrm{~nm}$. Fig. 3(a) shows how the reflected spectrum is gradually modified by the growing number of periods. Fig. 3(b) shows the reflected spectra for the first periods $(5,7,9,11,13$, and 15 periods).

It can be observed that a minimum number of layers-20 in this case-are needed to obtain a well-defined reflected peak, but increasing the number of layers above 40 does not lead to a noticeable improvement. The reflected optical power rises from 14.12 to $14.72 \mathrm{~dB}$ when the number of periods varies from 20 to 50 .
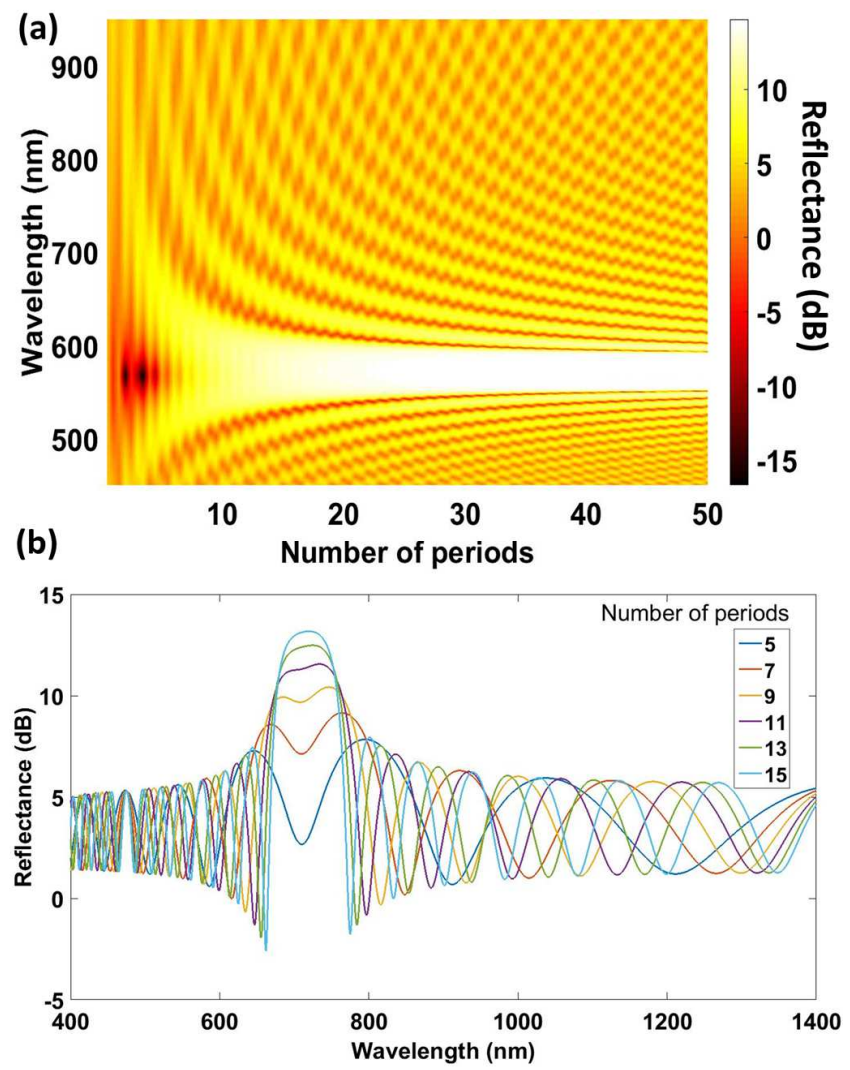

Fig. 3 (a) Evolution of the reflected spectrum as the number of periods increases, and reflected spectra for different numbers of periods: (b) 5, 7, 9, 11,13 , and 15 periods.

For a number of periods between 5 and 20, increasing the number of periods has a significant effect on the reflected optical power, making it possible to reduce the integration time of the spectrometer and hence to accomplish the measures faster. A good compromise between the optical spectrum obtained and the time spent to achieve it is reached within 50 bilayers.

\section{Thickness of periods}

Fig. 4 shows the effect of increasing the thicknesses of both layers while keeping their thicknesses equal to one another. For these results, the number of periods was kept constant and equal to 50 to obtain a well-defined peak. The RI of each layer was also kept constant (LRIL $=1.7$, HRIL $=1.87)$ and the thickness of both layers was swept from 70 to $150 \mathrm{~nm}$ in order to obtain the reflected peak inside the spectral range of interest. As the thickness increases, the reflected peak redshifts and widens. A second peak starts to appear for thicknesses greater than $110 \mathrm{~nm}$.
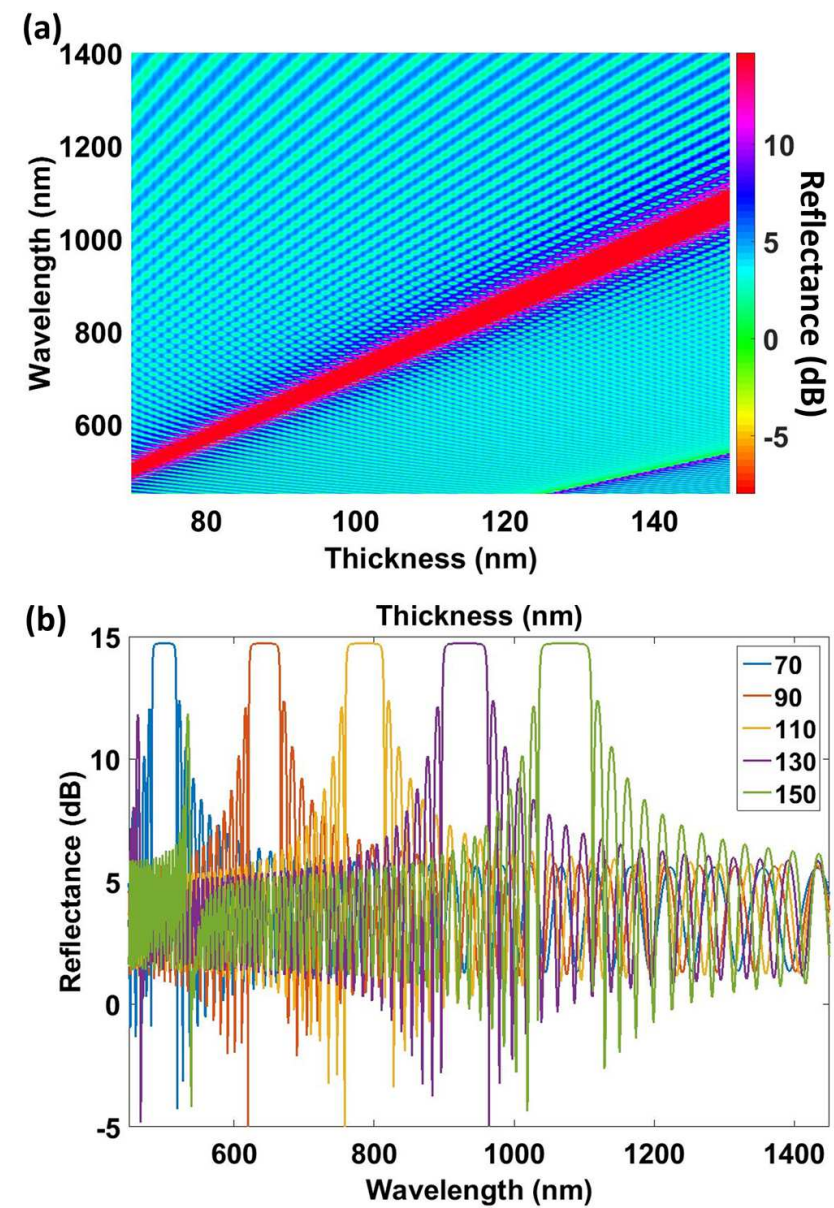

The effect of the thickness of the odd layers being different from the thickness of the even layers is similar to the effect of increasing the difference between RIs of the layers. The reflected peak widens and its location shifts to greater wavelengths as the difference between thicknesses increases.

\section{Extinction Coefficient}

Finally, the last factor that was studied was the effect of a non-zero absorption coefficient $(\alpha)$ on the results. The absorption coefficient depends on the material and also on the wavelength of light that is being absorbed [29]. The 
absorption coefficient is related to the extinction coefficient or the imaginary part of the RI $(\mathrm{k})$ and to the wavelength $(\lambda)$ by the following equation:

$$
\alpha=\frac{4 \pi k}{\lambda}(1)
$$

For these simulations, the number of periods was set to 50 and the real part of the RI of odd layers was set to 1.7 , while its extinction coefficient was swept from 0 to 0.2 . The RI of even layers was calculated as $10 \%$ greater than the RI of the odd layers. The simulated results are shown in Fig. 5.

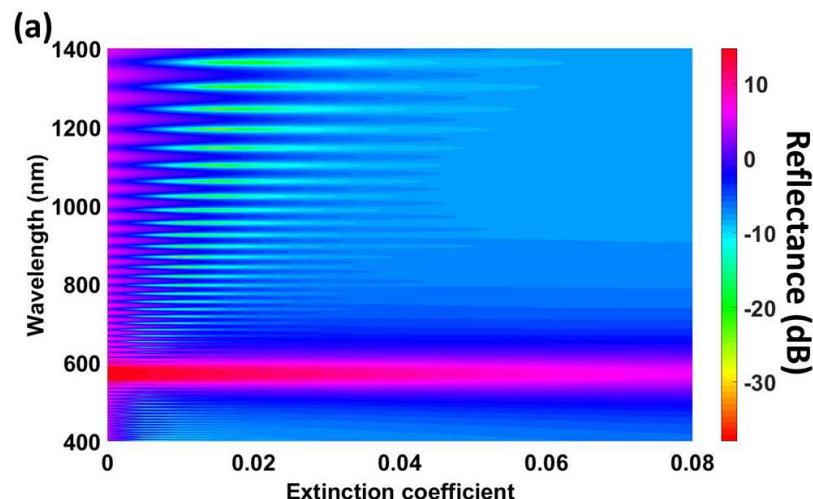

(b)

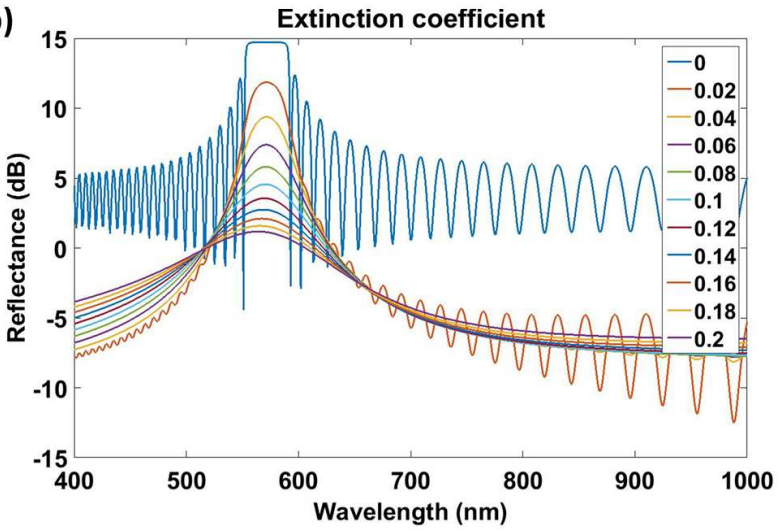

Fig. 5 (a) Evolution of the reflected spectrum as the extinction coefficient increases and (b) reflected spectra for different extinction coefficients.

For an extinction coefficient equal to zero, a flat band-pass filter would be obtained. Progressive attenuation of the reflected peak and the sidelobes occurs as the extinction coefficient increases. There is a quadratic relationship between the reflected optical power and the extinction coefficient. The reflected power decreases by $5 \mathrm{~dB}$ from $\mathrm{k}=0$ to $\mathrm{k}=0.035$ and another $5 \mathrm{~dB}$ is lost when the extinction coefficient reaches a value of 0.1 . For extinction coefficients greater than 0.2 , the reflected peak, as well as the sidelobes, tends to disappear and a flat reflected spectrum will be obtained for extinction coefficients greater than or equal to 0.5 (contrast lower than 2 $\mathrm{dB})$.

Material must be selected depending on the intended purpose of this optical structure. For the development of bandpass filters, a zero extinction coefficient should be selected, while for the development of optical fiber sensors working with the wavelength detection technique, a better defined peak might be better for the needs of the algorithm that tracks the wavelength where the reflected peak is located.

\section{EXPERIMENTAL RESULTS}

In this section, the experimental results of fabricating Bragg gratings on the end face of a standard multimode optical fiber are shown.

The cleaved end facet of an MMF pigtail was placed inside the vacuum chamber of a pulsed DC sputtering system (Nadetech) and connected by an optical feedthrough with the equipment mentioned before (see Fig. 1(a)). The optical fiber was centered with the cathode and the end facet of the optical fiber was placed parallel to the target surface. Three experiments were performed in order to obtain the reflected peak in the whole UV-Vis-NIR range that the HR-4000 spectrometer covers.

Two different thin film stacks were studied: stacks of indium oxide $\left(\mathrm{In}_{2} \mathrm{O}_{3}\right)$ and stacks of tin oxide $\left(\mathrm{SnO}_{2}\right)$. Changes in the RI for materials such as ITO [19] and tin oxide while changing the sputtering pressure have been previously studied [18], [30], and variations of around 5\% have been obtained. Therefore, changes in the RIs of indium oxide and tin oxide should be obtained just by changing the sputtering pressure.

The most effective method of obtaining significant changes in the RI of the material is by changing the pressure by means of an ON/OFF control. This control technique is applied to the valve that regulates the flow of argon in order to obtain a controlled increasing pressure rate of $0.2 \mathrm{bar} / \mathrm{s}$ and a decreasing pressure rate of $0.6 \mathrm{bar} / \mathrm{s}$. The power supply of the sputtering system was controlled in constant power mode ( $\sim 50$ $\mathrm{W})$.

\section{A. Indium oxide experiments}

The sputtering parameters for experiments done with indium oxide are presented in Table I. Two different devices (D1 and D2) have been developed in order to check the possibility of placing the reflected peak at different wavelengths. The number of periods is 60 , and the thickness of each layer has been estimated to be $64.5 \mathrm{~nm}$. Fig. 6 shows the evolution of the reflected spectrum for the device D1.

Table I. Sputtering parameters for experiments with indium oxide.

\begin{tabular}{|c|c|c|c|c|c|c|}
\hline & & P (mbar) & Voltage (V) & $\mathbf{I}(\mathbf{m A})$ & Time (s) & $\mathbf{N}$ \\
\hline \multirow{2}{*}{ D1 } & LRIL & $1 \times 10^{-1}$ & 237 & 200 & 30 & \multirow{2}{*}{60} \\
\hline & HRIL & $<2 \times 10^{-5}$ & 353 & 150 & 40 & \\
\hline \multirow{2}{*}{ D2 } & LRIL & $1.5 \times 10^{-1}$ & 160 & 150 & 60 & \multirow{2}{*}{40} \\
\hline & HRIL & $<2 \times 10^{-5}$ & 278 & 100 & 80 & \\
\hline
\end{tabular}

Fig. 6 shows how the reflected peak is formed at $439 \mathrm{~nm}$ with a full width at half maximum (FWHM) of $20 \mathrm{~nm}$. It can also be observed that the reflected peak starts to appear at the 20th layer and that further periods contribute to a reduction in the amplitude of the sidelobes. 

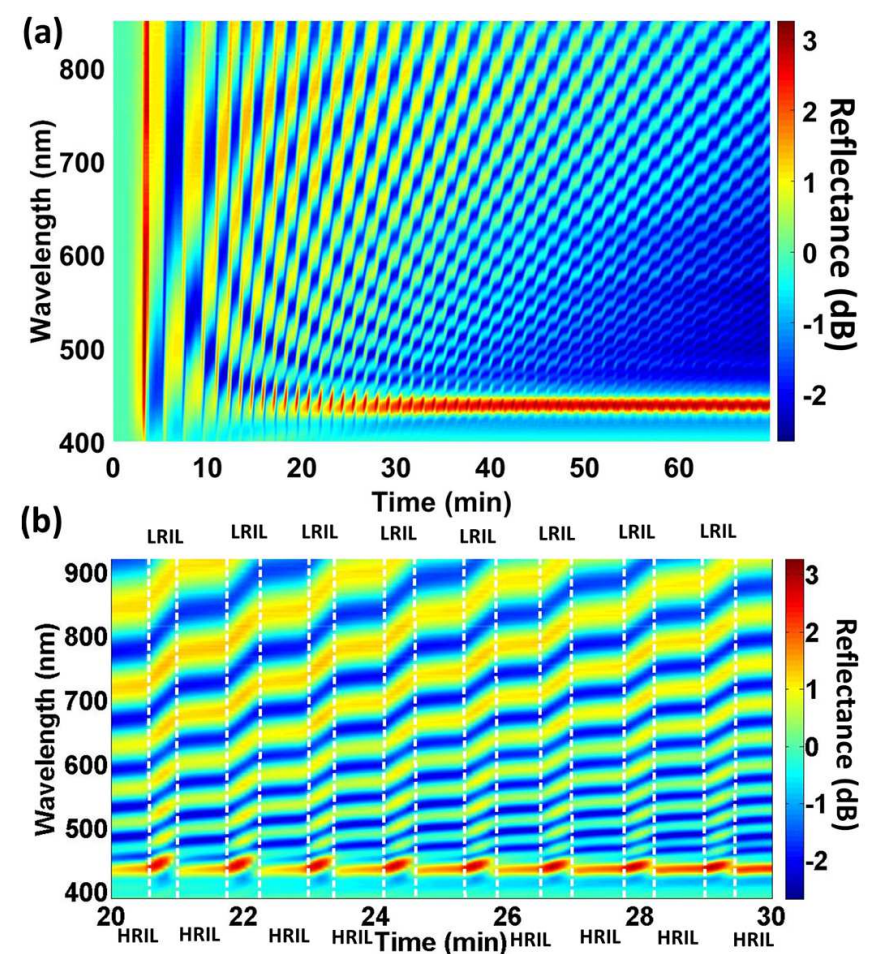

Fig. 6 (a) Evolution of the reflected spectrum collected during the sputtering process of indium oxide and (b) enlargement of the same figure, distinguishing between LRIL and HRIL.

In Fig. 7, several spectra for different numbers of layers are shown to give a better view of how the reflected peak is obtained. Sidelobes located closer to the reflected peak have the lowest amplitude, and a maximum contrast of $5 \mathrm{~dB}$ has been obtained.

Continuing with indium oxide, the experimental results of device D2, which consists of 40 periods, are shown in Fig. 8. The sputtering time has been doubled with respect to device D1, but the sputtering current has been reduced to $75 \%$. Now, the reflected peak is located at $639 \mathrm{~nm}$ and has a FWHM of 96 $\mathrm{nm}$ and a contrast of $5 \mathrm{~dB}$. Changes in the wavelength location are due to an increase in the thickness of each layer, while the increase of the FWHM is due to greater differences in the optical path between odd and even layers.
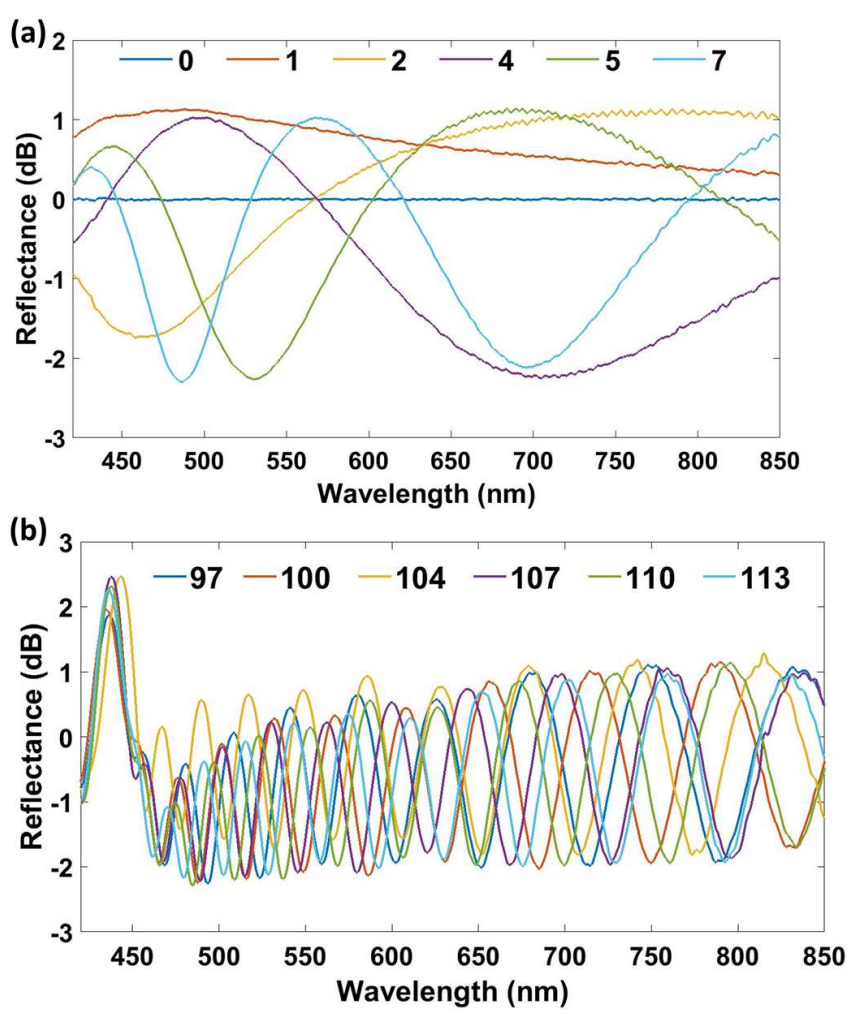

Fig. 7 Reflected spectra for (a) 0-7 layers and (b) 97-113 layers

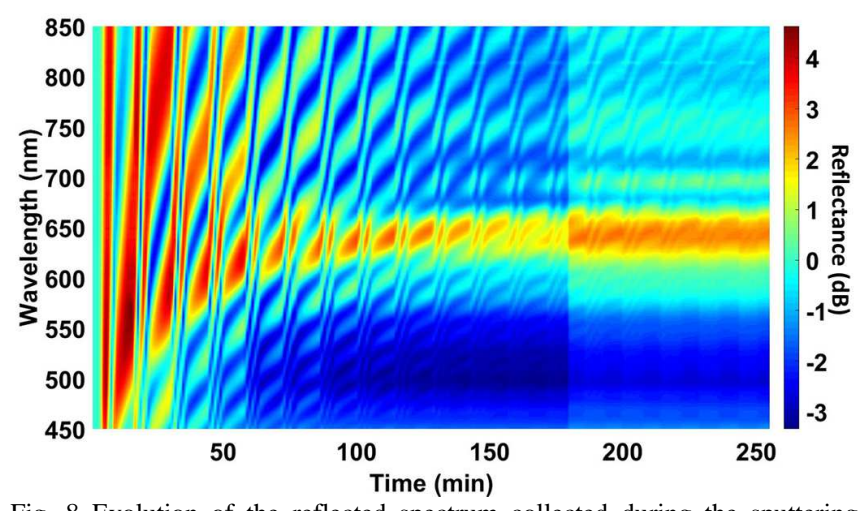

Fig. 8 Evolution of the reflected spectrum collected during the sputtering process of indium oxide with changes in the sputtering pressure for device D2

\section{B. Tin oxide experiment}

Now, the sputtered material was tin oxide and changes in its RI were obtained by the same method as was used in the previous section. The parameters used are summarized in Table II.

Table II. Sputtering parameters for experiment with tin oxide.

\begin{tabular}{|l|l|r|r|r|r|r|}
\hline \multirow{2}{*}{ D3 } & P (mbar) & Voltage $(\mathrm{V})$ & I (mA) & Time (s) & N \\
\cline { 2 - 7 } & HRIL & $<\times 10^{-1}$ & $\mathbf{1 6 0}$ & $\mathbf{1 5 0}$ & $\mathbf{3 5}$ & \multirow{2}{*}{21} \\
\hline
\end{tabular}

Fig. 9 shows the spectra collected during the fabrication of device D3, which has its reflected peak located at $828 \mathrm{~nm}$ and a FWHM of $49 \mathrm{~nm}$. The number of periods was limited to 21 and the thickness of each layer was estimated to be $109 \mathrm{~nm}$. 


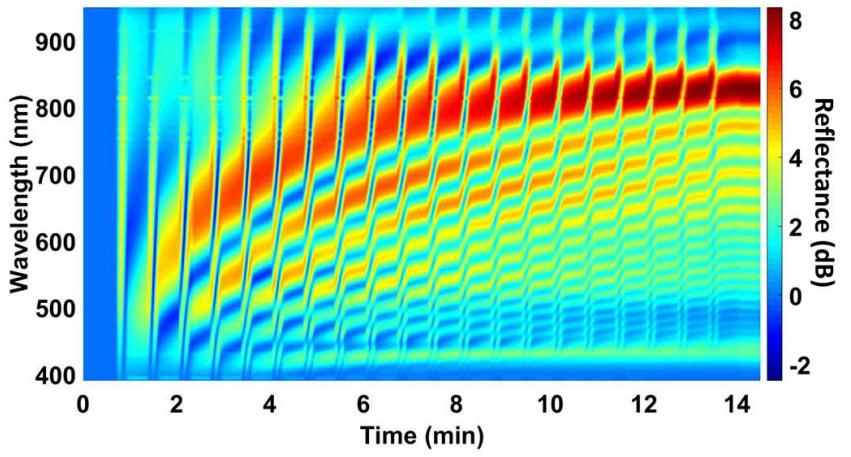

Fig. 9 Evolution of the reflected spectrum collected during the sputtering process of tin oxide.

This new Bragg grating has some differences from the previous one done with indium oxide. The sidelobes present greater amplitude because of the smaller number of periods. A reflected peak located at $828 \mathrm{~nm}$ with a FWHM of $49 \mathrm{~nm}$ has been obtained. The FWHM has increased with respect to the previous devices because it is placed at a greater wavelength and because of the optical constants of the material. The reflected power is also greater due to the increase of the real part of the RI of tin oxide compared to devices developed with indium oxide, as will be shown in the next section. A maximum reflectance of $8 \mathrm{~dB}$ with a maximum contrast of 10 $\mathrm{dB}$ has been obtained.

\section{COMPARISON BETWEEN THEORETICAL AND EXPERIMENTAL RESULTS}

Finally, a comparison between the experimental and simulated spectra was made. The simulated spectra were obtained by following the same procedure as the one used in the "Theoretical Analysis" section. Starting from values of optical constants and sputtering deposition rates obtained previously and following an iterative method based on the least squares method, the optimum parameters for fitting the theoretical and experimental data were found. For devices developed with indium oxide, the following parameters were used: The RI of the optical fiber was set to 1.5 and the number of periods was 60 for device D1 and 40 for device D2. The RI of even layers was set as $1 \%$ greater than the RI of odd layers. The thickness of each layer was set to $64.5 \mathrm{~nm}$ for device D1 and $93 \mathrm{~nm}$ for device D2. The average real part of the RI of odd layers was set to 1.6939 and an average extinction coefficient of 0.0253 was used for device D1, while the average real part of the RI of the odd layers was set to 1.7164 and an average extinction coefficient of 0.0347 was used. The RI of even layers was set as $4 \%$ greater than that of the odd layers.

For the device developed with tin oxide, the following parameters were used: The number of periods was 21 , the average RI of odd layers was set to 1.8558 , and an average extinction coefficient of 0.0334 was used. The RI of even layers was set as $3.5 \%$ greater than the RI of odd layers. The thickness of each layer was $109 \mathrm{~nm}$. A comparison between the simulated and experimental spectra achieved is shown in
Fig. 10, and the parameters obtained from simulations are summarized in Table III.

Table III. Estimated parameters for experiments extracted from simulations and reflected peak characteristics.

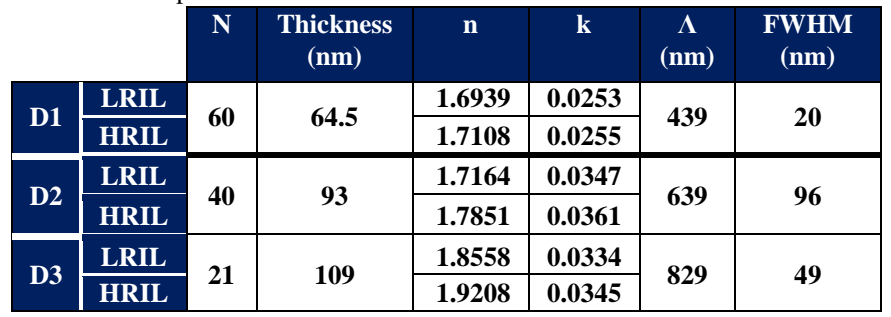

Optical constants extracted from the simulated results are in good agreement with the real values obtained by ellipsometry [19], [25], [26] or by other methods [18], as greater values of the RI of tin oxide were obtained compared to indium oxide.
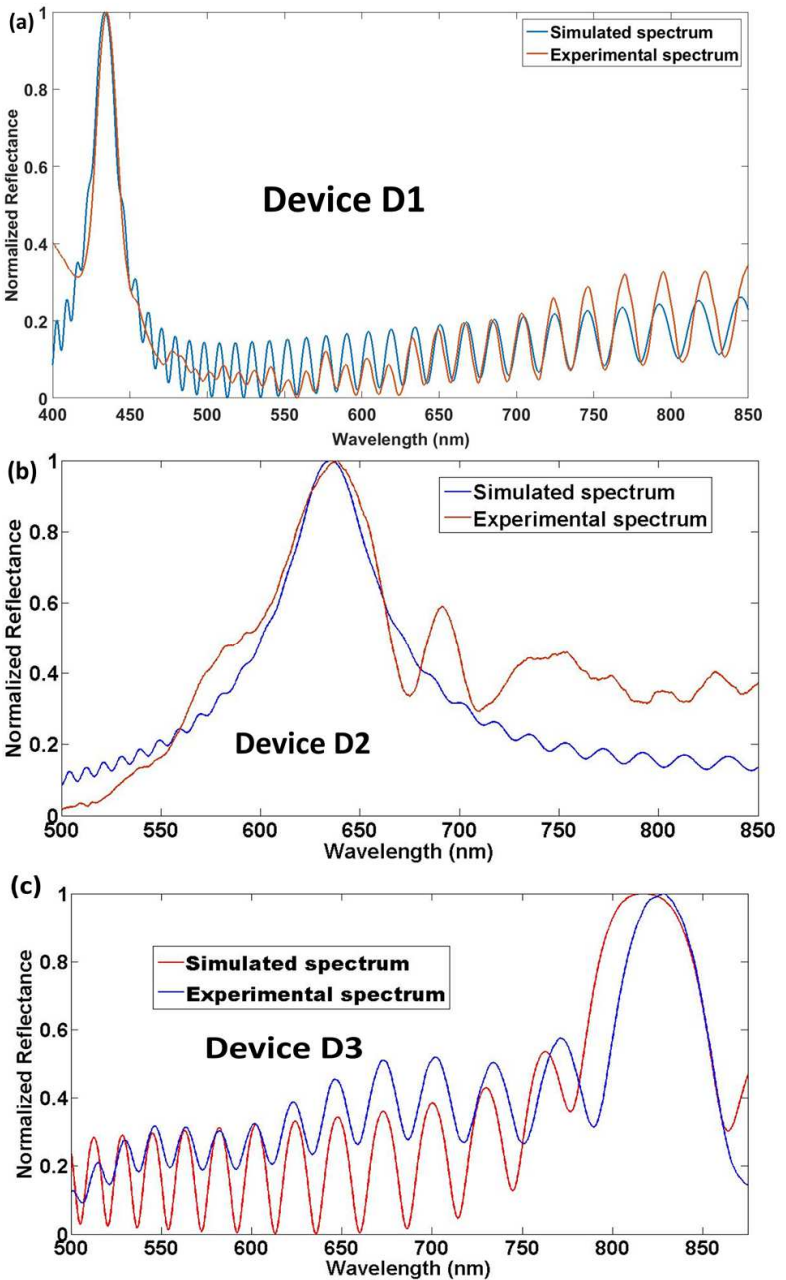

Fig.10 Comparison between experimental and simulated spectra of devices (a) D1 and (b) D2, both developed with indium oxide, and (c) for device D3 developed with tin oxide. 


\section{CONCLUSION}

This work has demonstrated the fabrication of a Bragg grating on the end facet of a standard MMF by means of sputtering using only one material and one sputtering gas. Changing the sputtering pressure is sufficient to obtain significant changes in the RI of the sputtered material. This procedure can be easily automated and controlled and it has some advantages over other methods: the fabrication time is reduced, it is possible to control the FWHM of the resonance, and only one material is needed. These changes make it possible to obtain a reflected peak located at $439 \mathrm{~nm}$ with a FWHM of just $20 \mathrm{~nm}$ with indium oxide and a reflected peak at $829 \mathrm{~nm}$ with a FWHM of $49 \mathrm{~nm}$ when working with tin oxide. This optical structure can be used for the development of wavelength tunable filters or optical fiber sensors.

The main factors affecting the optical spectrum obtained have also been analyzed. These factors are the relationship between RIs, the thickness of each layer, and the imaginary part of the RI. The difference between real parts of the RI of each layer should be greater than $2 \%$ to obtain a distinguishable reflected peak. Greater differences would increase the width of the reflected peak. The imaginary part of the RIs should be lower than 0.2 to have a clearly observable peak. Increasing the value of the extinction coefficient leads to progressive attenuation of the reflected peak. Finally, it has been proven that the location of the reflected peak depends on the optical path of each layer. The theoretical and experimental results are in good agreement.

\section{REFERENCES}

[1] H. Adachi, "1 - Thin films and nanomaterials," in Handbook of Sputtering Technology, 2012, pp. 3-39.

[2] H. N. Rogers, "High-temperature sputtered coatings for optical fiber," Proc. SPIE, vol. 1791, pp. 122-128, 1993.

[3] P. Sanchez, C. R. Zamarreño, M. Hernaez, I. R. Matias, and F. J. Arregui, "Optical fiber refractometers based on lossy mode resonances by means of $\mathrm{SnO} 2$ sputtered coatings," Sensors Actuators, B Chem., vol. 202, pp. 154-159, 2014.

[4] J. Ascorbe, J. M. Corres, F. J. Arregui, and I. R. Matias, "Magnetic field sensor based on a single mode-multimode-single mode optical fiber structure," in 2015 IEEE Sensors, 2015, pp. 1-4.

[5] J. Ascorbe, J. M. Corres, F. J. Arregui, and I. R. Matias, "Low voltage transducer based on the changes in the wavelength of the attenuation band," in IEEE Sensors 2014 Proceedings, December 2014, pp. 1912-1915.

[6] J. Ascorbe, C. Sanz, J. M. Corres, F. J. Arregui, I. R. Matias, and S. C. Mukhopadhyay, "High sensitivity extrinsic Fabry-Pèro interferometer for humidity sensing," in 2015 9th International Conference on Sensing Technology (ICST), 2015, pp. 143-146.

[7] J. S. Zeakes, K. A. Murphy, A. Elshabini-Riad, and R. O. Claus, "Modified extrinsic Fabry-Perot interferometric hydrogen gas sensor," Lasers Electro-Optics Society Annual Meeting 1994. LEOS '94 Conference Proc. IEEE, 1994, vol. 2, pp. 235-236'.

[8] A. Szeghalmi, M. Helgert, R. Brunner, F. Heyroth, U. Gösele, and M. Knez, "Atomic layer deposition of $\mathrm{Al} 2 \mathrm{O} 3$ and $\mathrm{TiO} 2$ multilayers for applications as bandpass filters and antireflection coatings," Appl. Opt., vol. 48, no. 9, pp. 1727-1732, 2009.

[9] M. F. Schubert, F. W. mont, S. Chhajed, D. J. Poxson, J. K. Kim, and E. F. Schubert, "Design of multilayer antreflection coatings made from co-sputtered and low-refractive index materials by genetic algorithm," Opt. Exp., vol. 16, no. 8, p. 5290-5298, 2008. $\mathrm{Y} . \mathrm{Tu}, \mathrm{Y}-\mathrm{H} . \mathrm{Qi}$, and S.-T. Tu, "Fabrication and thermal characteristics of multilayer metal-coated regenerated grating sensors for high-temperature sensing," Smart Mater. Struct., vol. 22, no. 7 , p. 75026,2013 .

[11] C. Huang, D. Lee, J. Dai, W. Xie, and M. Yang, "Fabrication of high-temperature temperature sensor based on dielectric multilayer film on sapphire fiber tip," Sensors Actuators A Phys., vol. 232, pp. 99-102, 2015.

[12] C. Huang, W. Xie, D. Lee, C. Qi, M. Yang, M. Wang, and J. Tang, "Optical fiber humidity sensor with porous $\mathrm{TiO} 2 / \mathrm{SiO} 2 / \mathrm{TiO} 2$ coatings on fiber tip,” IEEE Photonic Tech. L., vol. 27, no. 14, pp. 1495-1498, 2015.

[13] F. J. Arregui, I. R. Matias, K. L. Cooper, and R. O. Claus, "Fabrication of microgratings on the ends of standard optical fibers by the electrostatic self-assembly monolayer process.," Opt. Lett., vol. 26, no. 3, pp. 131-133, 2001.

[14] I. Pavlichenko, A. T. Exner, M. Guehl, P. Lugli, G. Scarpa, and B. V. Lotsch, "Humidity-enhanced thermally tunable $\mathrm{TiO} 2 / \mathrm{SiO} 2 \mathrm{Bragg}$ stacks," J. Phys. Chem. C, vol. 116, no. 1, pp. 298-305, 2012.

[15] S. G. Ilchenko, R. A. Lymarenko, and V. B. Taranenko, "Metalmultilayer-dielectric structure for enhancement of s- and p-polarized evanescent waves," Nanoscale Res. Lett., vol. 11, no. 1, p. 42, 2016.

[16] M. Hála, R. Vernhes, O. Zabeida, J. E. Klemberg-Sapieha, and L. Martinu, "Reactive HiPIMS deposition of SiO2/Ta2O5 optical interference filters," J. Appl. Phys., vol. 116, no. 21, 2014.

[17] A. Kumar, V. K. Sharma, D. Kumar, and A. Kapoor, "Integrated optic TE/TM pass polarizers using resonant coupling between ITO thin film lossy modes and dielectric waveguide modes," Opt. Commun., vol. 291, pp. 247-252, 2013.

[18] B. Stjerna, E. Olsson, and C. G. Granqvist, "Optical and electrical properties of radio frequency sputtered tin oxide films doped with oxygen vacancies, F, Sb, or Mo," J. Appl. Phys., vol. 76, no. 6, pp. 3797-3817, 1994.

[19] Y. S. Jung, "Spectroscopic ellipsometry studies on the optical constants of indium tin oxide films deposited under various sputtering conditions," Thin Solid Films, vol. 467, no. 1-2, pp. 3642, 2004.

[20] K. O. Hill and G. Meltz, "Fiber Bragg grating technology fundamentals and overview," J. Light. Technol., vol. 15, no. 8, pp. 1263-1276, 1997.

[21] K. O. Hill, B. Malo, F. Bilodeau, D. C. Johnson, and J. Albert, "Bragg gratings fabricated in monomode photosensitive optical fiber by UV exposure through a phase mask," Appl. Phys. Lett., vol. 62, no. 10, pp. 1035-1037, 1993.

[22] D. Z. Anderson, V. Mizrahi, T. Erdogan, and A. E. White, "Production of in-fibre gratings using a diffractive optical element," Electron. Lett., vol. 29, no. 6, p. 566, 1993.

[23] W. Yuan, A. Stefani, and O. Bang, "Tunable polymer fiber Bragg grating (FBG) inscription: Fabrication of dual-FBG temperature compensated polymer optical fiber strain sensors," IEEE Photonics Technol. Lett., vol. 24, no. 5, pp. 401-403, 2012.

[24] J. Dai, M. Yang, X. Yu, and H. Lu, "Optical hydrogen sensor based on etched fiber Bragg grating sputtered with $\mathrm{Pd} / \mathrm{Ag}$ composite film," Opt. Fiber Technol., vol. 19, no. 1, pp. 26-30, 2013.

[25] J. Ascorbe, J. M. Corres, I. R. Matias, and F. J. Arregui, "High sensitivity humidity sensor based on cladding-etched optical fiber and lossy mode resonances," Sensors Actuators, B Chem., vol. 233, pp. 7-16, 2016.

[26] N. Paliwal and J. John, "Lossy mode resonance (LMR) based fiber optic sensors: A review," IEEE Sensors J., vol. 15, pp. 5361-5371, May 2016.

[27] P. Yeh, A. Yariv, and C.-S. Hong, "Electromagnetic propagation in periodic stratified media'. I. General theory," JOSA, vol. 67, no. 4, p. $438,1977$.

[28] I. Del Villar, I. Matias, F. Arregui, and R. Claus, "Analysis of onedimensional photonic band gap structures with a liquid crystal defect towards development of fiber-optic tunable wavelength filters.," Opt. Express, vol. 11, no. 5, pp. 430-436, 2003.

[29] A. S. Blazev, "Crystalline silicon photovoltaic technologies," in Photovoltaics for Commercial and Utilities Power Generation, Fairmont Press, 2013.

[30] Khalaf, M. K., Al-Tememee, N. A., Ibrahim, F. T., \& Hameed, M. A, "Crystalline structure and surface morphology of tin oxide films grown by DC-reactive sputtering," vol. 4, no. 2, pp. 516-519, 2014. 